\title{
SARS-CoV2 envelope protein: non-synonymous mutations and its consequences
}

\author{
Sk. Sarif Hassan ${ }^{\mathrm{a}, *}$, Pabitra Pal Choudhury ${ }^{\mathrm{b}}$, Bidyut Roy $^{\mathrm{c}}$ \\ ${ }^{a}$ Department of Mathematics, Pingla Thana Mahavidyalaya, Maligram 721140, India \\ ${ }^{b}$ Applied Statistics Unit, Indian Statistical Institute, Kolkata 700108, West Bengal, India \\ ${ }^{c}$ Human Genetics Unit, Indian Statistical Institute, Kolkata 700108, West Bengal, India
}

\begin{abstract}
In the NCBI database, as on June 6, 2020, total number of available complete genome sequences of SARS-CoV2 across the world is 3617 . The envelope protein of SARS-CoV2 possesses several non-synonymous mutations over the transmembrane domain and (C)-terminus in $0.414 \%$ of these 3617 genomes. The C-terminus motif DLLV has been changed to DFLV and YLLV in the proteins QJR88103 (Australia: Victoria) and QKI36831 (China: Guangzhou) respectively, which might affect the binding of this motif with the host protein PALS1. Keywords: Envelope protein; SARS-CoV2; COVID-19; Non-synonymous mutations;
\end{abstract}

\section{Introduction}

The present pandemic of the Severe Acute Respiratory Syndrome (COVID-19) is caused by the RNA virus SARS-CoV2 which is characterized by its rapid mutations up to a million times higher than that of their hosts [1]. There are several mutations happened in various proteins of the SARS-CoV2 over a short period of virus to escape host immunity [5, 6]. So, such variability and evolution are enabling the virus to develop drug resistance [1]. Among various proteins of SARS-CoV2, spike(S), envelope (E), membrane(M) and nucleocapsid $(\mathrm{N})$ are the four structural proteins which form the outer layer of the coronavirus and they help in assembling and releasing new copies of the virus within human cell [7].

The $\mathrm{CoV}$ envelope (E) protein is the smallest among the four structural proteins involved in several aspects of the virus life cycle, such as assembly, budding, envelope formation, and pathogenesis [7]. However, the molecular mechanism involving $\mathrm{E}$ in pathogenesis is not yet clearly understood. Notably, this protein interacts with other structural proteins such as membrane(M), nucleocapsid $(\mathrm{N})$ and other accessory proteins viz. ORF3a, ORF7a and host cell proteins [8]. Envelope protein of SARS-CoV2 is 76 amino acids long and it possesses three important domains viz. (N)-terminus, transmembrane domain (TMD) and (C)-terminus (Fig.1). The (C)-terminal domain of envelope protein in SARS-CoV2 binds to human PALS1, a tight junctionassociated protein, which is essential for the establishment and maintenance of epithelial polarity in mammals

\footnotetext{
${ }^{*}$ Corresponding author

Email addresses: sarimif@gmail.com (Sk. Sarif Hassan), pabitrapalchoudhury@gmail.com (Pabitra Pal Choudhury), broy@isical.ac.in (Bidyut Roy)
} 
9].

\begin{tabular}{|c|c|c|c|c|}
\hline N-TERMINAL & TRANSMEMBRANE & & MINA & \\
\hline 10 & 30 & 40 & 60 & 70 \\
\hline
\end{tabular}

Figure 1: Amino acid sequence and domains of the envelope protein of SARS-CoV2 [7]

Figure 1 Legend: Red and blue colors are representing hydrophobic and hydrophilic amino acid, respectively.

There are four mutations including one deletion have been found in the envelope protein of SARS-CoV2 with reference to that of the SARS-CoV1. The alignment is given in Fig.2.

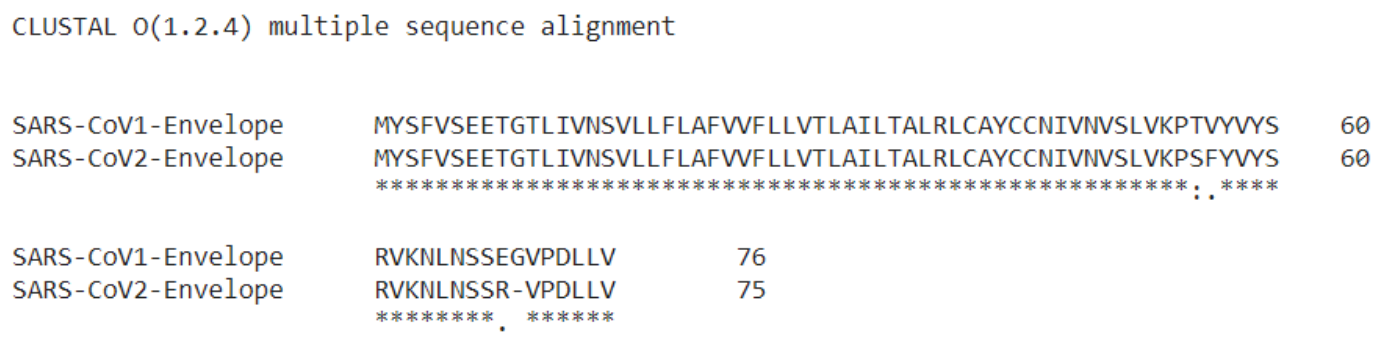

Figure 2: Clustal alignment of the envelope protein of SARS-CoV1 and SARS-CoV2

The Fig.2 legends: Mutations in (C)-terminus domain in the envelope protein of SARS-CoV2 are T55S, V56F, E69R (the mutation of an amino acid $A_{1}$ to an amino acid $A_{2}$ is denoted by $A_{1} p A_{2}$ where $p$ denotes location in the reference amino acid sequence) and the deletion mutation $G$ at the 70th position with respect to the reference envelope protein of SARS-CoV1.

The envelope protein of SARS-CoV possesses three important domains viz. (N)-terminus, transmembrane domain (TMD) and (C)-terminus. It is reported that the C-terminus domain of the envelope protein contains the motif DLLV (i.e. PDZ domain of the virus E-protein) which binds to the host cell PALS1 protein to facilitate infection $[9,10]$. In this present study, non-synonymous mutations in the envelope protein of SARSCoV2 across 3617 SARS-CoV2 genomes (as on 6th June, 2020), have been identified and accordingly their probable consequences are presented.

In this present study, non-synonymous mutations over the envelope protein of SARS-CoV2 across the available 3617 SARS-CoV2 genomes (as on 6th June, 2020), have been found and accordingly their probable

\section{Methods}

From the NCBI virus database, all the protein sequences of 3617 SARS-CoV2 genomes are fetched. Then the amino acid sequences of envelope protein of SARS-CoV2 are exported in fasta format using file oper- 
ations through Matlab. These sequences (fasta formatted) are blasted using Clustal-Omega and found the mismatched and from there mutations and their associated positions are accounted [1].

\section{Results}

Among these virus genomes from 3617 patients; there are 2588 were from USA, 372 were from Asia, 287 were from Europe, 365 were from Oceania and 5 were from Africa. Here, we present the non-synonymous mutations of the envelope protein over the available 3617 SARS-CoV2 genomes (Table 1). Note that the mutation of an amino acid $A_{1}$ to an amino acid $A_{2}$ is denoted by $A_{1} p A_{2}$ where $p$ denotes location in the reference amino acid sequence. In the Table 1 , also the changes of the R-group of each amino acid according to the mutations are presented. The Table-1 depicts the following:

Table 1: Non-synonemous mutation in the envelope protein of SARS-CoV2

\begin{tabular}{lccccc}
\hline Protein-ID & Geo-location & Mutation & Domain & Transition of R-group & Collection date \\
\hline QJA42107 & USA: VA & A36V & TMD* & Hydrophobic to Hydrophobic & $2020-04$ \\
QJQ84222 & USA: KENNER, LA & L26F & TMD & Hydrophobic to Hydrophobic & $04 / 04 / 20$ \\
QHZ00381 & South Korea & L37H & TMD & Hydrophobic to Hydrophilic & $2020-01$ \\
QJS53352 & Greece: Athens & L39M & TMD & Hydrophobic to Hydrophobic & $20 / 03 / 20$ \\
QJR88103 & Australia: Victoria & L73F & C-terminus & Hydrophobic to Hydrophobic & $22 / 03 / 20$ \\
QKE45838 & USA: CA & P71L & C-terminus & Hydrophobic to Hydrophobic & $30 / 04 / 20$ \\
QKE45886 & USA: CA & P71L & C-terminus & Hydrophobic to Hydrophobic & $30 / 04 / 20$ \\
QKE45898 & USA: CA & P71L & C-terminus & Hydrophobic to Hydrophobic & $30 / 04 / 20$ \\
QKE45910 & USA: CA & P71L & C-terminus & Hydrophobic to Hydrophobic & $30 / 04 / 20$ \\
QJE38284 & USA: CA & P71L & C-terminus & Hydrophobic to Hydrophobic & $28 / 03 / 20$ \\
QIU81527 & USA: WA & P71L & C-terminus & Hydrophobic to Hydrophobic & $19 / 03 / 20$ \\
QKG87268 & USA: Massachusetts & S68F & C-terminus & Hydrophobic to Hydrophobic & $01 / 04 / 20$ \\
QKG88576 & USA: Massachusetts & S68F & C-terminus & Hydrophobic to Hydrophobic & $06 / 04 / 20$ \\
QKI36831 & China: Guangzhou & D72Y & C-terminus & Hydrophilic to Hydrophobic & $27 / 02 / 20$ \\
QKI36855 & China: Guangzhou & S68C & C-terminus & Hydrophilic to Hydrophobic & $25 / 02 / 20$ \\
\hline
\end{tabular}

*TMD: transmembrane domain

- In less than $0.5 \%$ of the SARS-CoV2 genomes, the envelope protein possesses the missense mutations as adumbrated in the Table 1 . There are ten different mutations where the R-group property changes only in the case of the protein QHZ00381 for the mutation L37H in the TMD of the envelope protein.

- TMD was also observed to be conserved over the SARS-CoV1 and COV2 genomes, the protein sequences of QJA42107 (USA: VA), QJQ84222(USA: KENNER, LA), QHZ00381(South Korea) and QJS53352(Greece: Athens) possess four mutations A36V, L26F, L37H and L39M, respectively, in the TMD of the envelope protein. Change in the R-group property from Hydrophobic to Hydrophilic in the virus TMD from South Korea may affect the function of the envelope protein.

- The motif ' $D L L V^{\prime}$ has been changes to 'DFLV' and 'YLLV' in the proteins QJR88103 (Australia: Victoria) and QKI36831 (China: Guangzhou) due to the mutations L73F and D72Y respectively. These mutations having changes in the motif ' $D F L V^{\prime}$ might affect in binding this motif to the PALS1 of the host and accordingly may influence replication and/or infectivity of the virus. 
QKI36855 from China: Guangzhou respectively. Note that the mutation of the amino acid S to F keeps the R-group unchanged while that of the amino acid S to $\mathrm{C}$ changes the R-group Hydrophilic to Hydrophobic. This would possibly make changes in protein functions and interactions.

65

\section{Concluding remarks}

Among all the proteins associated to the novel RNA virus, some accessory proteins such as ORF6, ORF7b, ORF8, ORF10 contain least number of missense mutation as reported in various articles [12, 13, 14]. And same is true for envelope protein, E. We find $0.414 \%$ of the SARS-CoV2 genome contains eight different types of mutations in TMD and C-terminus of the envelope protein. Mutated E-protein might affect replication and propagation of the SARS-CoV2 as has been observed in cases of SARS-CoV and MERS-CoV in mouse model. Potential studies have also shown that vaccine against the E-protein mutated viruses can reduce the infectivity in mouse model.

\section{Author Contributions}

SH conceived the problem. SH determined the mutations. SH, PPC, BR analysed the data and result. SH

\section{Conflict of Interests}

The authors do not have any conflicts of interest to declare.

\section{References}

[1] M. Pachetti, B. Marini, F. Benedetti, F. Giudici, E. Mauro, P. Storici, C. Masciovecchio, S. Angeletti,

M. Ciccozzi, R. C. Gallo, et al., Emerging sars-cov-2 mutation hot spots include a novel rna-dependent-rna polymerase variant, Journal of Translational Medicine 18 (2020) 1-9.

[2] K. Xu, B.-J. Zheng, R. Zeng, W. Lu, Y.-P. Lin, L. Xue, L. Li, L.-L. Yang, C. Xu, J. Dai, et al., Severe acute respiratory syndrome coronavirus accessory protein $9 \mathrm{~b}$ is a virion-associated protein, Virology 388 (2) (2009) 279-285.

[3] S. S. Hassan, P. P. Choudhury, B. Roy, S. S. Jana, Missense mutations in sars-cov2 genomes from indian patients (May 2020). doi:10.31219/osf.io/2wm8h

[4] S. S. Hassan, P. P. Choudhury, P. Basu, S. S. Jana, Molecular conservation and differential mutation on orf3a gene in indian sars-cov2 genomes, BioRxiv (2020).

[5] Y. Li, X. Yang, N. Wang, H. Wang, B. Yin, X. Yang, W. Jiang, The divergence between sars-cov-2 and 90 ratg13 might be overestimated due to the extensive rna modification, Future Virology (0) (2020). 
[6] M. L. DeDiego, L. Pewe, E. Alvarez, M. T. Rejas, S. Perlman, L. Enjuanes, Pathogenicity of severe acute respiratory coronavirus deletion mutants in hace-2 transgenic mice, Virology 376 (2) (2008) 379-389.

[7] D. Schoeman, B. C. Fielding, Coronavirus envelope protein: current knowledge, Virology journal 16 (1) (2019) 69.

[8] R. McBride, M. Van Zyl, B. C. Fielding, The coronavirus nucleocapsid is a multifunctional protein, Viruses 6 (8) (2014) 2991-3018.

[9] K.-T. Teoh, Y.-L. Siu, W.-L. Chan, M. A. Schlüter, C.-J. Liu, J. M. Peiris, R. Bruzzone, B. Margolis, B. Nal, The sars coronavirus e protein interacts with pals1 and alters tight junction formation and epithelial morphogenesis, Molecular biology of the cell 21 (22) (2010) 3838-3852.

[10] T. Beuming, L. Skrabanek, M. Y. Niv, P. Mukherjee, H. Weinstein, Pdzbase: a protein-protein interaction database for pdz-domains, Bioinformatics 21 (6) (2005) 827-828.

[11] F. Madeira, Y. M. Park, J. Lee, N. Buso, T. Gur, N. Madhusoodanan, P. Basutkar, A. R. Tivey, S. C. Potter, R. D. Finn, et al., The embl-ebi search and sequence analysis tools apis in 2019, Nucleic acids research 47 (W1) (2019) W636-W641.

[12] S. Chatterjee, Understanding the nature of variations in structural sequences coding for coronavirus spike, envelope, membrane and nucleocapsid proteins of sars-cov-2, Envelope, Membrane and Nucleocapsid Proteins of SARS-CoV-2 (March 28, 2020) (2020).

[13] T. H. Tan, T. Barkham, B. C. Fielding, C.-F. Chou, S. Shen, S. G. Lim, W. Hong, Y.-J. Tan, Genetic lesions within the 3a gene of sars-cov, Virology journal 2 (1) (2005) 51.

[14] A. Pekosz, S. R. Schaecher, M. S. Diamond, D. H. Fremont, A. C. Sims, R. S. Baric, Structure, expression, and intracellular localization of the sars-cov accessory proteins $7 \mathrm{a}$ and $7 \mathrm{~b}$, in: The Nidoviruses, Springer, 2006, pp. 115-120. 